\title{
Research
}

\section{Energies Alterations and Chakras' Energies Deficiencies in Patient with Ulcerative Colitis}

\author{
Huang WL* \\ Medical Acupuncture and Pain Management Clinic, Franca, São Paulo, Brazil
}

${ }^{*}$ Correspondence to: Huang Wei Ling; Infectious disease, General Practice, Nutrition, Acupuncture and Pain Management Specialist, Medical Acupuncture and Pain Management Clinic, Franca, São Paulo, Brazil; Tel: 551637212437; Fax: 551637028540; E-mail: weilingmg@gmail.com

Received: Nov 28 ${ }^{\text {th }}$, 2020; Accepted: Jan 1 $1^{\text {st }}, 2021$; Published: Jan 21 $1^{\text {st }}, 2021$

Citation: Huang WL. Energies alterations and Chakras' energies deficiencies in patient with ulcerative colitis. Gastro Open A Open J. 2021; 2(1): 35-42. doi: 10.33169/gastro.GOAOJ-2-108

\begin{abstract}
Introduction

Ulcerative Colitis (UC) is a chronic, idiopathic inflammatory disease that affects the colon, most commonly afflicting adults aged 30 years to 40 years, resulting in disability. It is characterized by relapsing and remitting mucosal inflammation, starting in the rectum and extending to proximal segments of the colon. In traditional Chinese medicine (TCM), the invasion of external pathogenic factors causing Damp-Cold and Damp-Heat, inadequate diet and violent emotions are the source of the disease.
\end{abstract}

\section{Purpose}

The aim of this study is to demonstrate that patients with ulcerative colitis have energies alterations and chakras' energies deficiencies as underline causes.

\section{Methods}

A case report of a 32 years-old female patient with diagnosis of ulcerative colitis ten years ago (2010) and a very difficult childhood when her mother died before the disease began. The UC symptoms started with a bloated belly and excessive intestinal gas. She has been treated by proctologist doctor and was receiving Sulfasalazine medications but she was willing to have another type of treatment without using so many medications. She searched for traditional Chinese medicine treatment and the doctor did the diagnosis of Yin, Yang, Qi deficiency and Heat retention and was submitted to the radiesthesia procedure to see how her internal organs energy was. The result of this measurement was that all her six chakras were without energy (rated one out of eight) with the exception of the seventh chakra that was normal (rated in eight). The treatment consisted in Chinese dietary counseling, auricular acupuncture with apex ear bloodletting and replenishment the chakras energies using homeopathies according to Constitutional Homeopathy of the Five Elements Based on Traditional Chinese Medicine and crystal based medications.

\section{Results}

The patient showed a significant improvement after the treatment performed.

\section{Conclusion}

The conclusion of this study is that patients with ulcerative colitis have energies imbalances and chakras' energies deficiencies as demonstrated in this case report. The corrections of these energies imbalances is important to treat the root of the problem that are the energies imbalances and chakras' energies deficiencies and not just the symptoms presented by the patient.

Keywords: Ulcerative colitis; Energy; Chakras; Diet; Traditional Chinese medicine; Acupuncture; Homeopathy; Crystal-based medications. 


\section{INTRODUCTION}

Ulcerative colitis is a chronic, idiopathic inflammatory disease that affects the colon, most commonly afflicting adults aged 30 years to 40 years and resulting in disability. It is characterized by relapsing and remitting mucosal inflammation, starting in the rectum and extending to proximal segments of the colon. UC usually affects the distal colon, rectal mucosa, submucosa, and it can involve until the terminal ileum. The clinical manifestations are mainly recurrent mucopurulent bloody stool, abdominal pain, diarrhea, and combined systemic presentations to different degrees. The aim of therapy is to induce and maintain clinical and endoscopic remission. Colectomy is needed in up to $15 \%$ of patients with ulcerative colitis. ${ }^{1-2}$

In Western medicine, amino salicylates are the main choice of treatment for mild to moderate ulcerative colitis, topical and systemic steroids can be used to treat ulcerative colitis flares, while immunosuppressant and biological drugs are used in moderate to severe disease. ${ }^{1}$

The etiology of ulcerative colitis in Western medicine remains unknown. The genetic factor must be valued, since there is a greater family incidence in the general population. Psychological factors are considered strong contributors and precipitants of the disease in most cases, even if they cannot be considered causative agents. ${ }^{2}$

The diagnosis of clinical manifestations of UC lesions under colonoscopy exam, the application of pathological examinations, endoscopic mucosal staining techniques, and magnification endoscopy are increasing the scientific rigor of diagnosis. ${ }^{2}$

In traditional Chinese Medicine, emotional factors may be the cause triggering attacks of ulcerative colitis. The invasion of external Humidity, Cold and Heat, leading to Damp-Cold and Damp-Heat alterations, inadequate diet, violent emotions and constitutional deficiencies are the sources of the disease. ${ }^{2-3}$

Constitutional deficiencies usually refer to Spleen and Kidney deficiencies. Invasion of exterior pathogenic factors refers to Damp Heat in the Large Intestine and Damp-Cold in the Spleen caused by unbalanced diet high in raw food or cold injures the Spleen and Stomach can obstruct their functions in transforming and transporting food and nutrients. ${ }^{3}$

\section{PURPOSE}

The purpose of this article is based on the thoughts of Hippocrates, the father of Medicine, who says that it is very important to take into consideration the oldest medicines before the medicine currently used. This study pretends to demonstrate that patients with ulcerative colitis have energies alterations and chakras' energies deficiencies. The corrections of these energies imbalances through changing dietary habits according to Chinese dietary nutrition, acupuncture with apex ear bloodletting and replenishing the energies of the chakras' energies centers using highly diluted medications according to the theory Constitutional Homeopathy of Five Elements based on Traditional Chinese Medicine and crystal based medications are important tools to treat these kind of patients with this diagnosis.

\section{METHODS}

The methods used integrated a literature review of the ulcerative colitis articles in Western medicine and traditional Chinese medicine indexed in PubMed, and a case report of a 32 years-old female patient with this diagnosis.

\section{Case Report}

C.S, 32 years-old female patient, works as a nurse. She presented a diagnose of ulcerative colitis from ten years ago (2010). The ulcerative colitis symptoms started with a bloated belly and excessive gas in 2010 . She noticed at that time that her stools were loose, with mucus and blood, some days going to the bathroom over eight times with diarrhea. The patient also reported feeling severe cramps. The doctor requested a colonoscopy exam but the public health system in Brazil denied this exam and she did not perform it.

In 2011, the symptoms worsened when it was indicated again the colonoscopy exam. The result of this exam was that she was with ulcerative colitis, described to her as a chronic incurable disease, which she would have to intake medications throughout her whole life, even when the symptoms stop for a while in the remission phase. When the disease is in crisis, the patient need to ingest several drugs, such as antibiotics, corticosteroids, probiotics and vitamins. She goes to the bathroom several times during the day and her body cannot absorb the nutrients fast enough before she evacuates them, and for this reason, she losed a lot of weight and health.

When asked her about any emotional crisis, the patient said that she had a very difficult life throughout her childhood when her mother died when she was three years old and after this, she was taken care by several relatives, and always changing houses and her routine. She was very close to her sister as a child but her sister had to be separated from her to be cared for by another relative. Their separation was a bad mark for her. She cites that in addition of her problems during childhood without a family of her own, she also had recently broken up with a boyfriend and was suffering a lot. The author asked her about how ulcerative colitis was affecting her life, she told her that she felt very sadly because she got afraid of social events and leaving her house, because when the pain of the cramps comes, she has to rush to the bathroom. Her work life was also affected by these symptoms.

On her first medical appointment, the doctor did the diagnosis in traditional Chinese medicine of Yin, Yang and Qi deficiency with Heat retention, because she fells hot waves during the day (Yin deficiency) and fells cold in the extremities (Yang deficiency). She also fell excessive sweeting during the day ( $Q i$ deficiency) and bad breath and itchy in the skin (Heat retention). It was recommended to measure her seven chakras' using radiesthesia procedure and the result of this measurement was that all her six chakras were in the lowest level of energy (rated in one out of eight). Only the seventh chakra was in the normal level (rated in eight) (Figure 1).

Figure 1. Chakra's energies meridians deficient in energy with the exception of the seventh

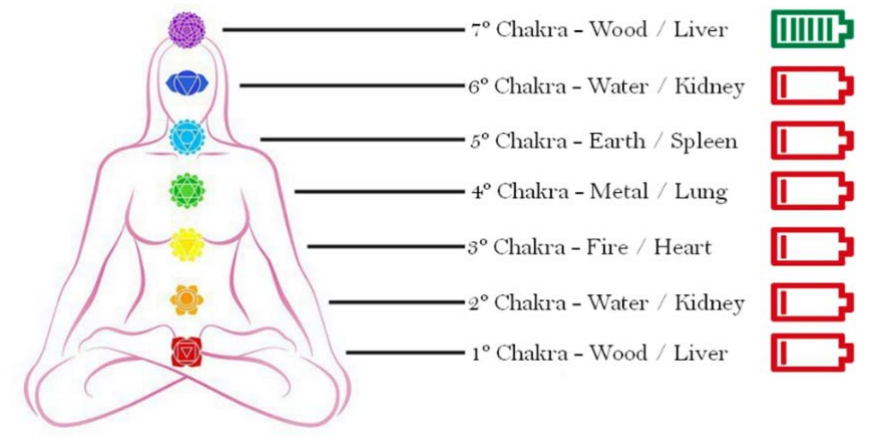


The first step of the treatment was changing her diet through Chinese nutritional therapy. She was counselled to avoid dairy products, raw food, cold beverages and sweets, to avoid the Spleen-pancreas deficiency. It was also recommended for the patient to avoid coffee; soda and matte tea, to avoid the Kidney deficiency. It was recommended to avoid chocolate, fried foods, honey, eggs, pepper, coconut, alcoholic beverages and melted cheese to avoid the formation of internal Heat.

The second step in the treatment was to balance the internal energies of Yin, Yang, Qi and Blood, using auricular acupuncture and it was necessary to take out the Heat retention through the use of apex ear bloodletting that was causing the ulcerative colitis inflammatory process and the bleeding from the stools (Figure 2).

Figure 2. Auricular Acupuncture Points

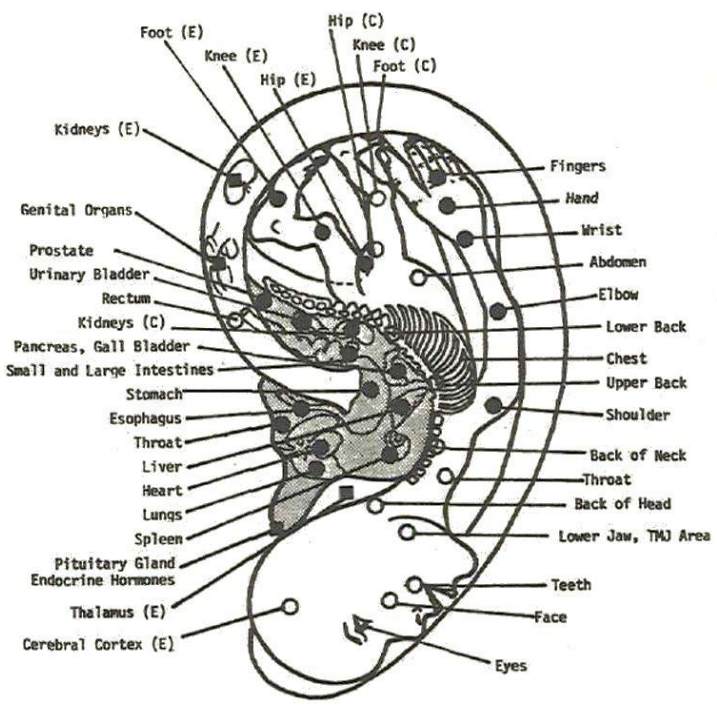

At last, the patient was prescribed treatment for replenishment of the chakras' energies with homeopathies, according to Constitutional Homeopathy of the Five Elements Based on Traditional Chinese Medicine, created by author and crystal based medications.

The following homeopathies were prescribed: Sulphur 30CHXX-20 (single dose), Calcarea carbonica 30CHXX-20 (single dose), Silicea 30CHXX-20 (single dose), Natrum muriaticum 30CHXX20 (single dose), Phosphorus 30CHXX-20. It was prescribed to be taken in a single dose with a hiatus of three days between the medications, necessarily on the sequence described. Two months later, a new sequence of treatment was started, with the same medications taken on the same order, but now with 200CHXX-20 instead of 30 CHXX-20. Two months later, a new sequence of treatment was started, as the others, with the same medications taken on the same order, now with 1000CHXX-20 instead of 200CHXX-20. Same order, now with 10000CHXX-20 instead of 1000CHXX-20. Two months later, a new sequence of treatment was started, as the others, with the same medications taken on the same order, now with 50000CHXX-20 instead of 10000CHXX-20.

These kind of medications are usually recommended to use for a period of one year or more, depending on the results of the new chakras' measurement after one year of treatment.

\section{RESULTS}

The patient showed a significant improvement after the treatment per- formed reducing the diarrhea symptoms and the abdominal pain reduced too. She affirmed that her mood improved and her energy to the daily activities and she was very happy with the results as the treatment were done in the deepest level of the energy disharmony, although she continues with the Western medicine medication.

In her latest visits, the patient shared she was often feeling breathless, which the doctor said that the symptoms were also related to her chakras' energies deficiencies, specifically by the deficiency of the second chakra represented by the Kidney in TCM.

In TCM reasoning, the Kidney meridian receives energy from the Lung meridian and when the Kidney is without energy, this transmission of energy is interrupted and can cause dyspnea symptoms.

This lack of breath was quickly dealt with the replenishment of the chakras' energies using highly diluted medications according to the theory Constitutional Homeopathy of Five Elements Based on Traditional Chinese Medicine when she was orientated to intake each medication on a daily basis, instead of each three days, because the patient was in a condition that needs urgent care to prevent further hospitalization.

\section{DISCUSSION}

Ulcerative Colitis (UC) is understood as a chronic inflammatory condition causing continuous mucosal inflammation of the colon without granulomas on biopsy, affecting the rectum and a variable extent of the colon in continuity, which is characterized by a relapsing and remitting course. $^{4}$

Colitis is the term best suited for the minority of cases where a definitive distinction between UC, Crohn's disease, or other cause of colitis cannot be made after the history, endoscopic appearances, histopathology of multiple mucosal biopsies and appropriate radiology have been taken into account. ${ }^{4}$

The first aim of medical management is to induce and maintain remission with the long-term goals of preventing disability, colectomy, and colorectal cancer. ${ }^{5}$

The treatment of ulcerative colitis is mainly done by Western medicine using amino salicylic acid preparations (salazosulfapyridine -SASP and aminosalicylic acid- ASA), steroid hormones, immunosuppressant's, and new biologics. ${ }^{6}$

The use of early biological drugs should be considered in patients admitted to hospital with acute severe ulcerative colitis, as well as in steroid-refractory ulcerative colitis. Once remission is induced, medications can be continued or added to maintain remission. ${ }^{5}$

To understand how the author did her reasoning to treat this patient presented in the case report, in the past years, the author has been publishing several studies, all aiming to establish the significance and effectiveness of an integral and individualized perspective, when treating patients for the most varied pathologies. ${ }^{7-8}$

This concept begins during the treatment of one particular patient in 2006. This patient was a 70-year-old male, who related pain in the legs and was diagnosed with Kidney-Yang deficiency, according to TCM. He received treatment with Chinese dietary counselling, acupuncture and auricular acupuncture associated with apex ear bloodletting. $^{7-8}$

When the treatment done, the pain in the legs diminished and 
the patient was subject to an appointment 30 days after. In this interview, the patient revealed that his eye pressure had also lowered, confirmed by his ophthalmologist. The doctor was not aware that the patient had glaucoma and he didn't report that he was being treated glaucoma in the last 40 years with no improvement of his condition that was always more than $40 \mathrm{~mm} \mathrm{Hg}$ in both eyes. With the processing performed, his intraocular pressure lowered to $17 \mathrm{~mm} \mathrm{Hg}^{7-8}$

This case became the cornerstone of the author studies in the field, trying to comprehend how the treatment based on the root of the problem could treat different diseases and symptoms simultaneously and using the same methods. ${ }^{7-8}$

To better explain how the author makes her reasoning, she always like to use the metaphor of the tree. The use of this metaphor is usually done to make a comparison of Western medicine from ancient medicines, such as traditional Chinese medicine. On the (Figure 3 ), you can see the root, trunk and several branches. Coming out of each branches, many leaves. In this representation, each medical specialty is represented by a branch, and the leaves of each branch represent the symptoms and diseases treated by each specialty. In this representation, Western medicine treats all the diseases in the leaf level. In this article, the author wants to explain that there is a necessity of correcting the energies imbalances that corresponds to the root level of this tree that is not visible by the naked eye. In this root, there are the Yin and Yang theory and the Five Elements theory. ${ }^{7-8}$

Figure 3. Schematic relationship between Western medicine and traditional Chinese medicine.

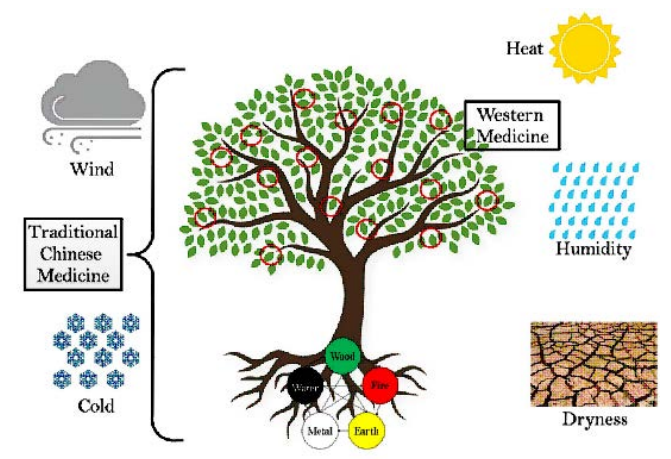

Yin and Yang are the two reverses forces that are believed to be present in all phoneme in the world. In traditional Chinese medicine, the Yin and Yang is the prime and major cornerstone to understand health, diagnosis and treatments. ${ }^{9-10}$

It is impossible to understand traditional Chinese medicine without understanding the main concepts of Yin and Yang. This concepts base everything in which traditional Chinese medicine believes in terms of treatment and cure of pathologies. Yin and Yang are energy forces that rule the world. Everything in the universe is composed by Yin and Yang, including our body. The Chinese wise men represented this complement of opposites by the archetypal pair, Yin and Yang, considering their dynamic interaction as the essence of all-natural phenomena and all human situations. Opposites are complementary. To be healthy, we aim for a state of equilibrium between Yin and Yang energies. If there are imbalances between these two energies, symptoms occur, and if not properly treated, consolidates the formation of disease, in this case, ul- cerative colitis (Figure 4$){ }^{11}$

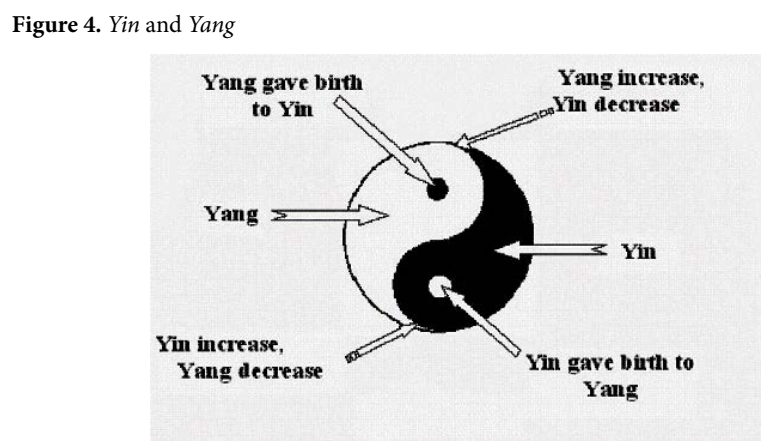

According to traditional Chinese medicine, the Yin and Yang energies are present and are important energies inside the body. We all have Yin and Yang energies, and to these energies flow inside the body, there is the necessity of $Q i$ and Blood. To be healthy, the patient need to be in a state of equilibrium between these four energies, as the author presented the necessity of treating these energies disharmonies to treat all the patient's symptoms at the same time, even if the doctor does not know that the patient had such symptom, as she presented this study at Acupuncture research conference that took place in the Harvard Medical School in Boston, USA, in 2015. The figure of the dynamic between these four energies are described in the (Figure 5). ${ }^{8}$

Figure 5. Yin, Yang, Qi and Blood.

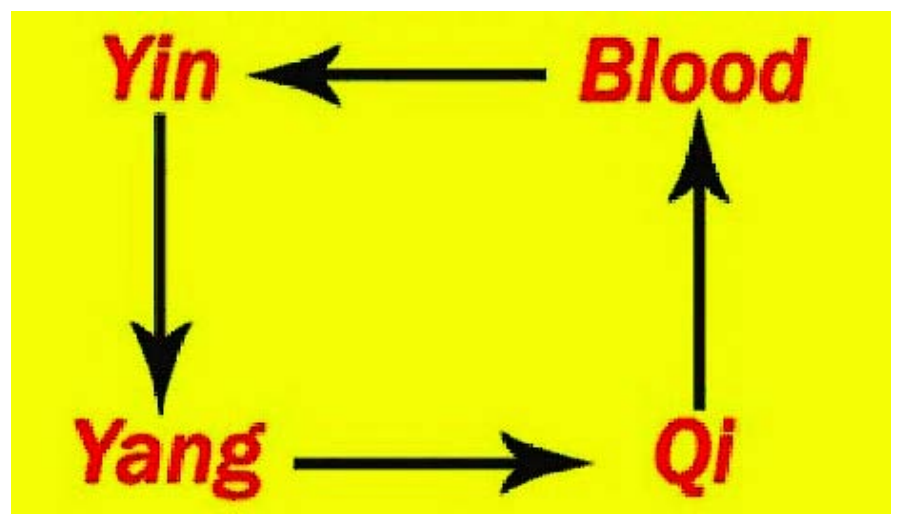

The Five Elements theory states that there are five elements present in everything in the world, including our bodies. These elements are Fire, Earth, Metal, Water and Wood (Figure 6). Inside our bodies, specific organs will represent these elements. These organs are considered the main organs, and they have extremely important functions in the well-functioning of the body. They are completely interconnected, as the main principle of the Five Elements theory is to understand the idea of cycle, as shown in the (Figure 6). ${ }^{10}$

The Wood element corresponds to the Liver. The Fire element corresponds to the Heart. The Earth element corresponds to the Spleen. The Metal element corresponds to the Lungs, and the Water element corresponds to the Kidney. ${ }^{10}$

These main organs will support (generation cycle) and control each other (control cycle) presented by the Five Elements, as demonstrated in the (Figure 6). ${ }^{10}$ 
Figure 6. Generations cycle and control cycle in the Five Elements theory

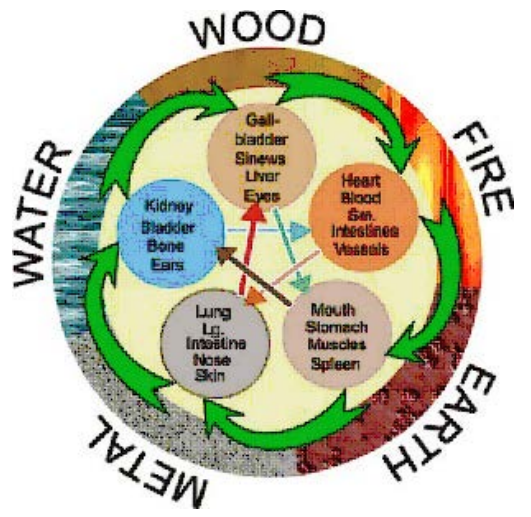

In the generation cycle, each element originates from the precedent element. In this cycle, Wood generates Fire, Fire generates Earth, Earth generates Metal, Metal generates Water and Water generates Wood. ${ }^{11}$

In the article The Geometry of Emotions: Using Chakra Acupuncture and 5-Phase Theory to Describe Personality Archetypes for Clinical Use written by Christopher R Chase, he associates the Five Elements theory with the chakras' energies centers in Ayurveda medicine. To publish this article and using this association explained by Christopher, the author is measuring the energy of the internal organs of the Five Elements using radiesthesia procedure in Ayurveda reasoning, as you can see in the (Figure 7$).{ }^{11}$

Figure 7. Chakra's energies centers and the correlation with the Five Elements and the five massive organs in TCM.

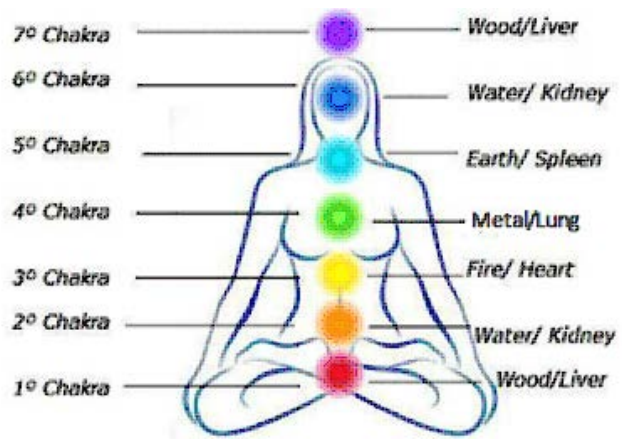

As there are seven chakras and five elements, the first and seventh correspond to Wood (Liver), the second and sixth correspond to Water (Kidney), the third to Fire (Heart), the fourth to Metal (Lung) and the fifth to Earth (Spleen). ${ }^{11}$

Each massive organ has a hollow organ as demonstrated in (Table 1). Liver or, first chakra, has the eye as external sensory organ, is responsible for vision, and has the Gall bladder as its hollow organ. Heart, or the third chakra, is responsible for the tongue, and subsequently language and has the Small intestine as its hollow organ. Spleen, or fifth chakra, represents the mouth, is responsible for taste, and has the Stomach as its hollow organ. Lungs, or fourth chakra, have the nose as external sensory organ, are responsible for the sense of smell, and have the Large intestine as its hollow organ. Kidney, or second chakra, rep- resents the ear, is responsible for the sense of hearing, and has the bladder as its hollow organ. In the case of the patient described in the case report, she has alterations in the Large intestine that is a hollow organ of the Lung, or fourth chakra. ${ }^{10-12}$

This information is important for the Western medicine physician, because the visible symptoms of external sensorial organs are just the tip of the iceberg. The cause, which is in the root, maybe found through this relation and is invisible by the naked eyes. In the case of the patient reported in this article, her symptoms were only the tip of the iceberg but the cause of the disease were in the deepest level, in the energy level, invisible by naked eyes. ${ }^{10-12}$

The author, through this case report, is demonstrating that the patient has no energy in all the chakras' energy centers but the chakra that is responsible for the formation of this disease is the disequilibrium in the fourth chakra. As the energy in this chakra was rated in one, in the lowest level of energy, this deficiency was leading to the formation of internal Heat in the Large intestine, because the Large intestine is the hollow organ that is coupled to the Lung, as demonstrated in Table 1.

Table 1. Five Elements, the five massive organs, and their correspondences

\begin{tabular}{l|l|l|l|l|l|} 
& \multicolumn{1}{|c|}{ Wood } & \multicolumn{1}{|c|}{ Flre } & \multicolumn{1}{|c|}{ Earth } & \multicolumn{1}{c|}{ Metal } & \multicolumn{1}{c|}{ Water } \\
\hline Orientation & East & South & Middle & West & North \\
\hline Season & Spring & Summer & Late Summer & Autumn & Winter \\
\hline Climate & Wind & Summer Heat & Dampness & Dryness & Cold \\
\hline Cultivation & Germinate & Grow & Transform & Reap & Store \\
\hline Yin Organ & Liver & Heart & Spleen & Lung & Kldney \\
\hline Yang Organ & Gall Bladder & Small Intestine & Stomach & Large Intestine & Bladder \\
\hline Orifice & Eye & Tongue & Mouth & Nose & Ear \\
\hline Tissues & Tendons & Vessels & Muscles & Skin \& Halr & Bones \\
\hline Emotions & Anger & Joy & Pensiveness & Grlef & Fear \\
\hline Colour & Blue/ Green & Red & Yellow & White & Black \\
\hline Taste & Sour & Bitter & Sweet & Pungent & Salty \\
\hline Voice & Shout & Laugh & Sing & Cry & Groan \\
\hline
\end{tabular}

In the case of this patient, her chronic emotional state of having sadness because of the constant problems after the dead of her mother since childhood leaded to the imbalance of the Lung meridian, that can be imbalanced by chronic state of sadness or grief, as demonstrated in the Table 1, and has the Large intestine as the hollow organ. The lack of energy in the chakras' centers, in this specifically case, in the fourth chakra, was causing formation of internal Heat in the hollow organ, that is the Large intestine, leading to the inflammatory process in this organ, and bleeding. ${ }^{10-12}$

In relation to nutrition, the author usually uses this aspect in all her treatments because she always follows what Hippocrates said: Make your food your medicine and your medicine your food. The point of view of Chinese nutritional therapy is different from Western nutritional therapy, because in first case, it is a study of the energy of each food leading to the patient's energy balance or imbalance. Unlike Western nutritional therapy, they study the part of chemical composition, biochemistry, vitamins, carbohydrates, proteins, fats etc. and usually don't take into account the energy part of each food. ${ }^{13-14}$

For this reason, it is of utmost importance, to have the knowledge of the energy of each food, leading to the energy imbalance of each individual, which will depend on the energy imbalance of each one, the 
treatment will be individualized. ${ }^{13-14}$

To achieve balance through diet, the author usually takes into account the balance state of the Spleen, Kidney and Liver. The Spleen is the door for entrance of energy, through absorbing the nutrients that the patient is ingesting, and if it is imbalanced, as demonstrated in the radiesthesia procedure, that the fifth chakra was in the lowest level of energy, this means that the food that the patient is ingesting is not absorbing adequately, and leading to less formation of Blood. This could be one of the causes that the remaining chakras were in the lowest level of energy, due to the lack of nutrients supply. ${ }^{13-14}$

The foods that usually imbalance the Spleen-pancreas meridian, or fifth chakra, are the ingestion of raw food, cold water, dairy products and sweets. In this case, the author usually advice the patients to eat boiled or grilled foods and drink luck warm water, to rebalance this meridian again. The energy of the water is Cold and when a Cold food enters the interior of the body, it generates the formation of an internal Heat, leading to the worsening of the formation of internal Heat in patients who already have internal Heat generated by the energy imbalances leading to ulcerative colitis symptoms. ${ }^{13-14}$

To reduce the energy imbalances in the Kidney meridian, responsible for the formation of Yin and Yang energy, the author orientates the patients to avoid coffee, soda and matte tea. This orientation is important because, as demonstrated in the radiesthesia procedure, the Kidney or second chakra was in the lowest level of energy too, and if the author don't orientate adequately this patient, the treatment could be in vain, because she was toning the meridian but reducing the energy but the wrong eating hobbits. The consequence of this lack of energy could form more internal Heat, worsening in this case, the ulcerative colitis symptoms. ${ }^{13-14}$

The last group of foods that the author usually asks the patients to avoid are: fried foods, chocolate, honey, eggs, melted cheese, coconut, alcoholic beverages and pepper. For example, when the patient ingests alcoholic beverages, in this case, beer, this drink has Hot energy, and when taken cold, it will cause the formation of internal Heat because the cold of the beer turns into Heat, forming more internal Heat, worsening the symptoms of ulcerative colitis. ${ }^{13-14}$

When internal Heat builds up, the patient's body in an attempt to eliminate this internal Heat can lead to the following symptoms: nasal bleeding; gingival bleeding; micro hematuria; abundant menstrual blood (prolonged in days of the cycle and for blood), bad breath; intestinal bleeding, etc. ${ }^{13-14}$

In the case of bleeding from this pathology, it is the manifestation of the internal Heat formation triggered by the energy deficiency of the patient, or generated by the wrong diet or emotional factors, leading to the formation of internal Heat (Figure 8). ${ }^{13-14}$

Figure 8. Yin, Yang, Qi, Blood and Heat retention.

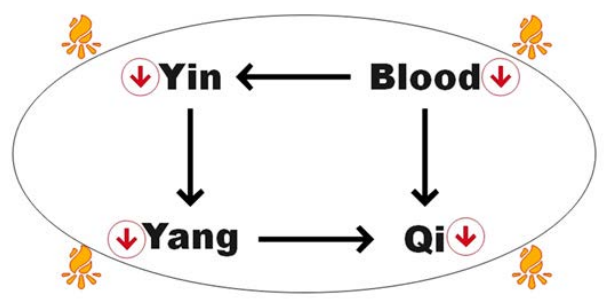

Therefore, in order to treat the bleeding of this pathology, in addition to removing the Heat formation by apex-ear bloodletting and reducing the foods that are generating the Heat formation directly, in the background, we have to treat the energies deficiencies that are generating the formation of Heat, as demonstrated by the results of radiesthesia procedure of the patient reported in this article. In this case, where all her six chakras were in the lowest level of energy, this energy deficiency was leading consequently to the formation of internal Heat, and causing the inflammatory process in the intestine. The bleeding process is considered the way that our body has to eliminate the excessive Heat formation. ${ }^{13-14}$

As each organ is dependent on another organ in the acquisition and transformation of energy, and one massive organ depends on the other, instead of treating the affected organ, as Western medicine does, all the organs that were energy deficient, diagnosed through energy measurement by radiesthesia, were treated. For example, in the case report, despite having ulcerative colitis, which was caused by the deficiency of the fourth chakra, this chakra depended on the energy of the fifth chakra. However, the fifth chakra depended on the energy of the third chakra - which also depended on the energy of the first chakra and which depended on the energy of the second chakra, as demonstrated in the Figure 9, in the generation cycle (Figure 10). ${ }^{13-14}$

Figure 9. Generation Cycle in the right and Control Cycle in the left.
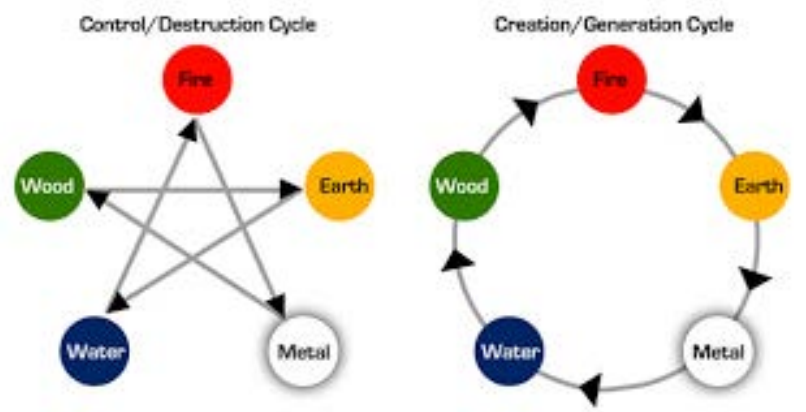

Figure 10. Chakras' energies deficiencies in ulcerative colitis patients, mainly the fourth chakra that has the Large intestine as the hollow organ

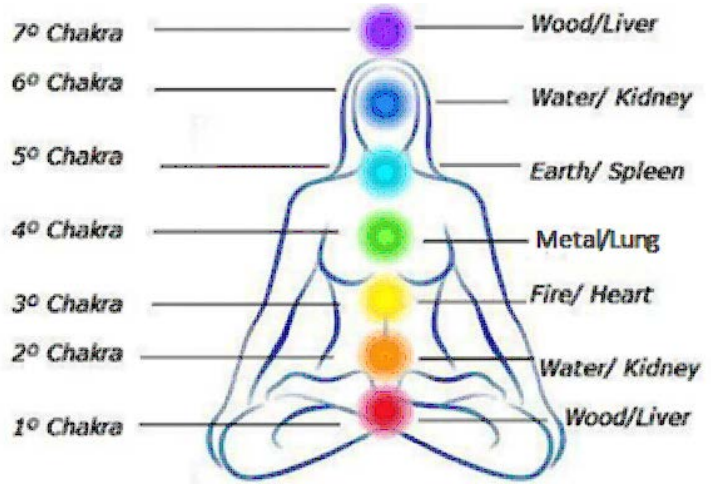

Homeopathies and crystal-based medications were used by the author to treat the patient with ulcerative colitis and they are shown in the (Table 2). ${ }^{10-11}$ 
Table 2. Homeopathies and crystal based medications used to replenish the chakras' energy meridians

\begin{tabular}{c|c|c|c}
\hline Chakras & Five Elements & Homeopathy Medications & Crystal-based medications \\
\hline $1^{\circ}$ Chakra & Wood $/$ Liver & Phosphorus & Garnet \\
\hline $2^{\circ}$ Chakra & Water $/$ Kidney & Natrium muriaticum & Orange calcite \\
\hline $3^{\circ}$ Chakra & Fire $/$ Heart & Sulphur & Rhodochrosite \\
\hline $4^{\circ}$ Chakra & Metal $/$ Lung & Silicea & Emerald \\
\hline $5^{\circ}$ Chakra & Earth $/$ Spleen & Calcarea carbonica & Blue Quartz \\
\hline $6^{\circ}$ Chakra & Water $/$ Kidney & Tone $2^{\circ}$ chakra & Sodalite \\
\hline $7^{\circ}$ Chakra & Wood $/$ Liver & Tone $1^{\circ}$ chakra & Tiger eye
\end{tabular}

In the article wrote by the author entitled Constitutional Homeopathy of the Five Elements Based on Traditional Chinese Medicine, published by Acta Scientific Medical Sciences on July 2020, the author describes how was her reasoning when she created this new theory using homeopathies medications and associating Chinese medicine thoughts. ${ }^{10}$

In the article entitled Why Are Diabetic Patients Still Having Hyperglycemia despite Diet Regulation, Ant glycemic Medication and Insulin? published by International Journal of Diabetes \& Metabolic Disorders on March 2019, the author described the crystal-based medications used in the treatment of replenishment of the chakras' energies centers and their properties and the type of each crystal are described in the Table $2 .{ }^{15}$

The author chooses to use highly diluted medications (homeopathies) instead of highly concentrated medications in this case, because the principals of Arndt Schultz law. ${ }^{15}$ The rule states that for all substances, small doses stimulate, moderate doses inhibit and overdoses kill. ${ }^{15}$ According to this law, the use of high-concentrated drugs will reduce the patient's vital energy that was already low, as demonstrated in the radiesthesia procedure described in the case report of this patient. ${ }^{16}$

The use of medication in high concentration usually used in the treatment of this kind of patients should be used with care, as they can cause a greater reduction in vital energy, leading to greater internal Heat formation generating more inflammatory process in the large intestine and leading to more bleeding in the stools (Figure 11). ${ }^{16}$

Figure 11. Arndt Schultz law

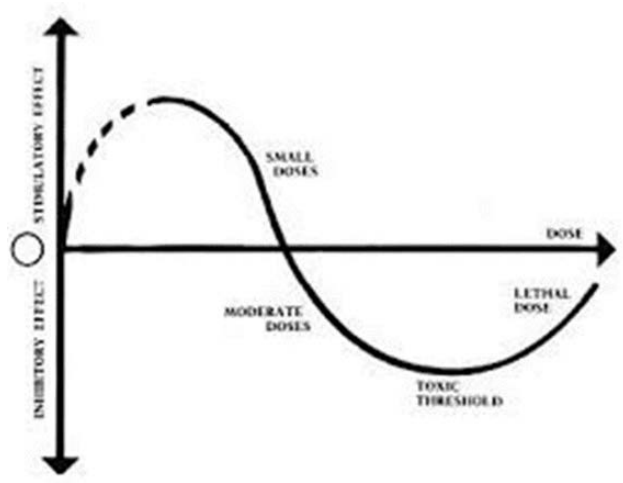

To better comprehend the physiopathology of the formation of the disease, the physician need to have these two medicines in the mind, to understand in the deepest level, the reason for the formations of the diseases including in this case, the emotional factors, the diet, the external pathogenic factors, that belongs to the TCM reasoning. The association of this two kinds of medicine (Western and traditional Chinese medicine) is the extreme importance to have a better outcome and results of the treatment of patients with ulcerative colitis (Figure 12). ${ }^{17-22}$

Figure 12. Metaphor of Western medicine and traditional Chinese medicine

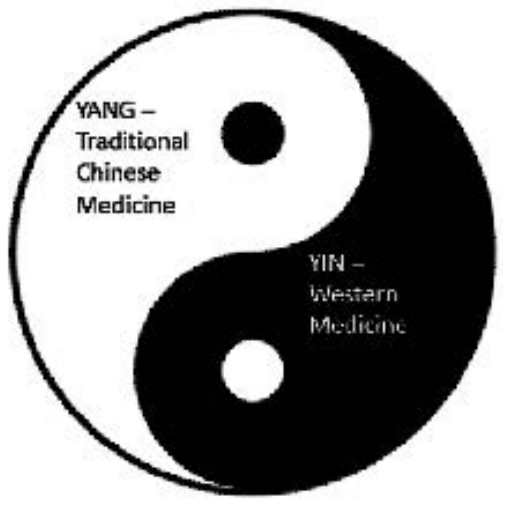

\section{CONCLUSION}

The conclusion of this study is that ulcerative colitis have energies imbalances of Spleen, Kidney, Liver and Lung leading to the formation of Heat retention. There are also chakras' energies deficiencies in this patient reported in this case report, that need to be corrected to treat in a deeper way, at the root of the problem and not just the symptoms.

For a better understanding of the causes of ulcerative colitis, it is necessary to understand what is happening in the root of the problem, that are the energies imbalances and chakras' energies deficiencies, which are invisible to the naked eye. The association of the teachings of Chinese medicine with Western medicine is essential for understanding in a deeper way and a more appropriate treatment.

\section{CONFLICTS OF INTEREST}

None.

\section{REFERENCES}

1. Ungaro R; Mehandru S; Allen P; Biroulet L; Colombel J. Ulcerative colitis. US National Library of Medicine National Institutes of Health. 2016 Dec. 1.

2. Gomes R. Fisiopatologia e tratamento por acupuntura da retocolite ulcerativa. Faculdade de educação, ciência e tecnologia -unisaude formação de especialistas em acupuntura. Brasília. 2008.

3. Chen J. Tackling Ulcerative Colitis with Herbs. Acupuncture Today. 2011; 12: 2 .

4. Stange E, Travis S, Vermeire S, Reinisch W, Geboes K. European evidence-based Consensus on the diagnosis and management of ulcerative colitis: Definitions and diagnosis. Journal of Crohn's and Colitis. 2008; 2: 1 .

5. Ungaro R, Mehandru S, Allen P, Biroulet L, Colombel J. Ulcerative colitis. US National Library of Medicine. 2016; 10(8): 925-33. doi: 


\subsection{3/ecco-jcc/jjw049}

6. Ji J, Huang Y, Wang X, Ma Z, Wu H, Im H, et al. Review of Clinical Studies of the Treatment of Ulcerative Colitis Using Acupuncture and Moxibustion. Gastroenterology Research and Practice. 2016; 3: 9248589. doi: $10.1155 / 2016 / 9248589$

7. Stanley J. TCM: External Causes of Disease. Available at 19-10-2018

8. Teixeira J. Nasal Septal Deviations: A Systematic Review of Classification Systems. Available at 19-10-2018.

9. Huang Wei Ling. How Do You Treat Back Pain in Your Practice? Part. 2. Medical Acupuncture. 2018; 30(1). doi: 10.1089/acu.2017.29070.cpl

10. Huang Wei Ling. Constitutional Homeopathy of the Five Elements based on Traditional Chinese Medicine. Acta Scientific Medical Sciences. 2020; 57-69.

11. Chase C. The Geometry of Emotions: Using Chakra Acupuncture and 5-Phase Theory to Describe Personality Archetypes for Clinical Use. National Center for Biotechnology Information. 2018 Aug.

12. Huang Wei Ling. Shoulder and Elbow Tendinitis as Initial Manifestation of Autoimmune Hepatitis. ARC Journal of Orthopedics. 2019; 4(2): 19-26. doi: 10.20431/2456-0588.0402004

13. Huang W L. What is the Cause of Language Impairment in Traditional Chinese Medicine and how can we treat it? J Clin Case Rep Trials. 2019; $2: 2$.
14. Ling HW. Is it Possible to Treat Community-Acquired and Nosocomial Infections with the Same Method, Without the Use of Antibiotics? J Appl Microb Res. 2019; 2(2): 1-13.

15. Huang Wei Ling. Why Are Diabetic Patients Still Having Hyperglycemia despite Diet Regulation, Antiglycemic Medication and Insulin? International Journal of Diabetes \& Metabolic Disorders. 2019.

16. Leeser O. Support of homeopathy by the Arndt-Schulz law. National Center for Biotechnology Information. 1953; Jul 31.

17. Craik E. The 'Hippocratic' Corpus. Routledge; 1ª Edição (17 dezembro 2014).

18. Yapijakis C. Hippocrates of Kos, The Father of Clinical Medicine, and Asclepiades of Bithynia, The Father of Molecular Medicine. Review. In Vivo. 2009; 23(4): 507-514.

19. Coimbra O. Semeando Luz: Sistema Cristais de Oz. Chiado Editora. 2017.

20. Volker Scheid. Traditional Chinese medicine-What are we investigating? The case of menopause. Complement Ther Med. 2007; 15(1-3): 54-68. doi: 10.1016/j.ctim.2005.12.002

21. Nan Lu. The Precepts of Traditional Chinese Medicine. Encyclopædia Britannica. Feb 02, 2009.

22. Maoshing NI. The Yellow Emperor's Classic of Medicine: The Essential Text of Chinese Health and Healing. Shambala, Boston. 